\title{
A LARGE-SCALE FLEXIBLE MOLDING TECHNOLOGY FOR PRODUCING BIOMIMETIC DRY ADHESIVES IN MUTLIPLE MATERIALS USING A COMMERICAL ACRYLIC MASTER
}

\author{
D. Sameoto ${ }^{1,2 *}$ and C. Menon ${ }^{2}$ \\ ${ }^{1}$ Mechanical Engineering Department, University of Alberta, Edmonton, Alberta, Canada \\ ${ }^{2}$ MENRVA Research Group, Simon Fraser University, Burnaby, British Columbia, Canada
}

\begin{abstract}
We present a novel fabrication method that uses $254 \mathrm{~nm}$ light from germicidal lamps to convert commercial acrylic substrates into master molds for biomimetic dry adhesives. These geckoinspired adhesives use appropriately shaped fibers to make intimate contact with surfaces and adhere using van der Waals interactions. While previous molding technologies for synthetic dry adhesives were limited by the size of the substrates used, our technology makes use of bulk acrylic, and is technically possible to scale up to dozens of square feet per master mold. Nearly any castable polymer may be converted to a dry adhesive using this technology.
\end{abstract}

\section{INTRODUCTION}

Biomimetic dry adhesives are inspired by the fibrillar structures found on the feet of geckos and certain spiders which allow the animals to adhere through van der Waals interactions. Synthetic versions of these materials are typically manufactured using polymers cast on nano and microscale molds. These molds have usually been based on silicon [1-2] or photoresists on silicon wafers [3-4] which severely limits the maximum size of adhesive sheets. Mushroom shaped fibers are desirable to produce significant normal adhesion forces, requiring either direct molding from undercut holes [5], or dipping methods [6-7] to produce overhanging caps on pre-formed fibers. While we have previously reported a photoresist based manufacturing technology that allows direct casting of mushroom shaped silicone dry adhesives [3-4], both the lifetime and size of the mold were limited. In the manufacturing technology that we are reporting in this paper, a master mold made of acrylic and SU-8 is used for casting negative silicone molds which are flexible and easily release other cured polymers. It is difficult to make other materials bond to silicones, making them an ideal choice for casting the final dry adhesives out of polyurethanes, epoxies and other curable polymers. Additionally, the bulk acrylic used as a substrate may be purchased in sizes up to 4'x8' providing an upper limit on the mold size far in excess to that of the largest silicon wafers. The qualities of the particular silicone chosen for making negative molds also permit the assembly of large molds from multiple individual sheets.

\section{FABRICATION PROCESS}

The direct photopatterning of polymethylmethacrylate (PMMA) using $254 \mathrm{~nm}$ light was originally conceived as a lowcost alternative to LIGA or e-beam lithography and later on was used for self-sacrificial polymer MEMS processing [8-9]. The sensitivity of PMMA to $254 \mathrm{~nm}$ light is extremely low, leading to lengthy exposure times ( $>5$ hours), but the relative low cost of this light source (used industrially as germicidal lamps) is attractive for exposing large areas. Germicidal lamps have previously been used to expose commercial acrylic (OPTIX ${ }^{\circledR}$ from Plaskolite) to directly form microfluidics channels [10]. Acrylic is primarily PMMA with some additives and can be an inexpensive alternative to silicon or SU-8 micromachining when directly photopatterned. The aspect ratio and feature sizes produced in OPTIX ${ }^{\circledR}$ were severely limited by the uncollimated $254 \mathrm{~nm}$ light source that was used (Stratalinker 2400 [10]). Our modified process is capable of producing higher aspect ratio structures, and more importantly, is capable of producing the overhanging structures that are critical for strongly adhering polymer dry adhesives. The basic process is outlined in Figure 1 and described below.
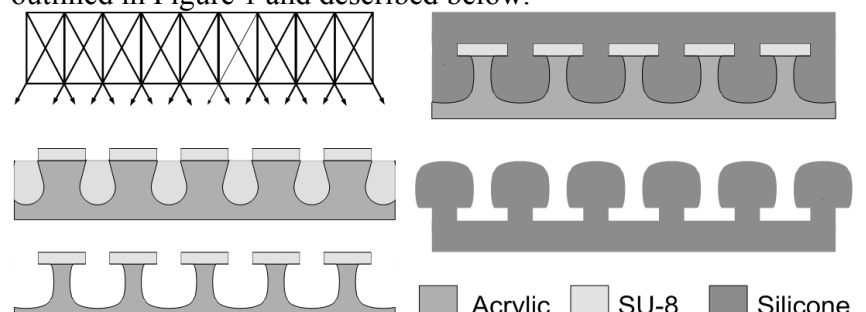

Acrylic $\square$ su-8 $\square$ Silicone

Figure 1. Schematic of the basic process steps used to convert commercial acrylic to dry adhesive molds. Partially collimated $254 \mathrm{~nm}$ light exposes commercial acrylic using a thin SU-8 layer as a mask. The acrylic is developed to produce fibers with thin overhanging SU-8 caps. The mold is replicated in silicone, and the negative silicone mold is ready to replicate the acrylic/SU-8 fibers in softer materials.

\section{Fabrication Steps}

- SU-8 2002 is spun on a commercial acrylic substrate (OPTIX ${ }^{\circledR}$ from Plaskolite) then prebaked in an oven to form a thin layer $(\sim 1.6 \mu \mathrm{m})$ on the acrylic surface.

- The SU-8 is exposed to i-line light from a standard UV aligner to define the fiber cap size and shapes.

- The SU-8 is post exposure baked for longer than 1 hour at $80^{\circ} \mathrm{C}$ to maximize the crosslinking, while keeping the acrylic well below the temperature at which it may warp or sag.

- The SU-8 is developed to form a hard mask on the acrylic

- The acrylic is exposed to $254 \mathrm{~nm}$ light with the presence of either a single level or dual level anti-scatter grid made from a plastic grating. The SU-8 is mostly opaque to $254 \mathrm{~nm}$ light and acts as a mask in perfect contact with the acrylic. Exposure to $254 \mathrm{~nm}$ light lowers the molecular weight of the acrylic through main chain scission, and increases its dissolution rate in developers.

- After exposure, the acrylic is developed using SU-8 developer at room temperature $\left(18{ }^{\circ} \mathrm{C}\right)$. A slightly lower selectivity of this solvent than the IPA: $\mathrm{H}_{2} \mathrm{O}$ developer used earlier [8-10] allows for better undercutting of SU-8 features. The final fiber shape depends on the exposure dose, anti-scatter grid design and development time.

- A high-strength, high temperature tolerant silicone (TC-5045 from BJB-Enterprises) is used to cast a negative mold from the acrylic/SU-8 master. This silicone has much higher tear strength than Sylgard ${ }^{\circledR} 184$ silicone, making it a more attractive option for large size, high durability molds.

- Casting of polyurethanes is completed using TC-5045 molds and the adhesion performance of different fibers is compared.

A custom $254 \mathrm{~nm}$ source is used to provide higher power exposures and consists of a stainless steel box containing $1225 \mathrm{~W}$ bulbs. A sample is placed on a battery powered turntable (AUTOArt 8" Rotary Display Turntable Stand) immediately under 
an anti-scatter grid and exposed through the anti-scatter grid while rotating. This rotation during exposure evens out the exposure dose and avoids shadowing specific areas. The anti-scatter grid is a plastic grating $12.5 \mathrm{~mm}$ thick with square holes $12.5 \times 12.5 \mathrm{~mm}$ in size arranged in a single or dual level configuration to make $1: 1$ or $2: 1$ aspect ratio grids. Although anti-scatter grids are quite common for reducing noise in X-ray imaging, they are not commonly used for UV light because a lot of light intensity is lost. However, a semi-collimated exposure can be completed over large areas at one time using this method. The effect of the anti-scatter grid is also assisted by the natural effect of Snell's Law as the UV passes from the air to the higher index of refraction acrylic. This angle enhancement is most apparent for the low aspect ratio grids, but still assists for 2:1 aspect ratio grid features (Figure 2).

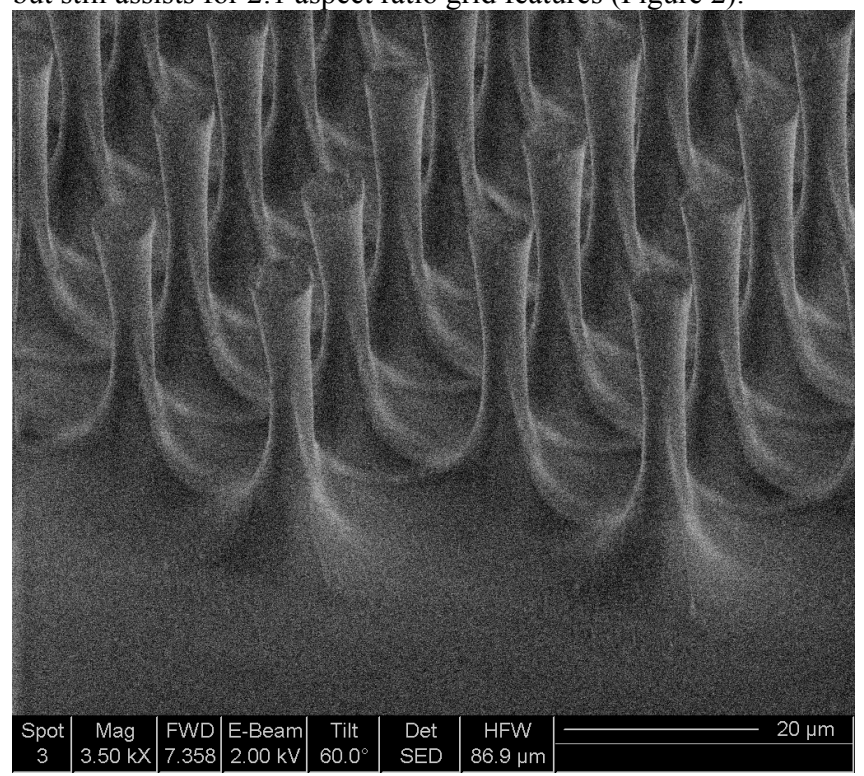

Figure 2. 4:1 aspect ratio acrylic fibers defined with a 2:1 aspect ratio anti-scatter grid. A metal mask rather than $S U-8$ defined fibers without caps.

\section{EXPERIMENTS}

A series of experiments were completed on two 5"x5" acrylic squares where the aspect ratio of the anti-scatter grid, the exposure time to $254 \mathrm{~nm}$ light and development time were varied. Circular SU-8 caps on a square array were defined with thickness of 1.6 $\mu \mathrm{m}$. Two different diameters of caps were defined, but the variations with best yield and adhesion behavior at this cap thickness were $12 \mu \mathrm{m}$ in diameter with a center to center spacing of $20 \mu \mathrm{m}$. The substrates were exposed to $254 \mathrm{~nm}$ light with different anti-scatter grid aspect ratios to four different doses. After exposure, development in SU-8 proceeded in 10 minute increments, after which the molds were rinsed and dried. The developed depth in wide open areas was measured and a negative mold was cast from TC-5045 to later make dry adhesives with the resulting geometry at each stage of development. Visual inspection was done at each stage as well to determine failure modes and estimate the yield of fibers for each process condition.

The TC-5045 silicone molds were cast with low-modulus polyurethane (ST-1060 from BJB Enterprises) that has been characterized by other researchers for use as a dry adhesive [1, 67]. This particular polyurethane has extremely high tear strength, reducing the likelihood of damage during demolding, but its modulus is relatively low ( $\sim 3 \mathrm{MPa})$ making fibers vulnerable to collapse if they are too tall or narrow. For higher aspect ratio fibers, stiffer polyurethane (WC-755 from BJB Enterprises) was also used to make dry adhesive structures. Its tensile modulus is approximately $100 \mathrm{MPa}$ and it has a listed Shore D hardness of 55 .

To test the relative adhesion performance of fibers produced using this process, a custom measurement system was used. This system consisted of a sensitive force probe (GSO-25 from Transducer Techniques) combined with a $6 \mathrm{~mm}$ diameter hemispherical sapphire tip (Edmund Optics, NT49-556), attached to a linear stage controlled by custom software. Adhesion vs. preload was recorded for multiple trials at a pull speed of $5 \mu \mathrm{m} / \mathrm{s}$.

\section{RESULTS}

We have found that the degree of crosslinking in the SU-8 appears to be a critical factor in its adhesion to acrylic and is best controlled by the use of exposure dose because the baking temperature is limited by the thermal tolerance of the acrylic. Initial experiments indicated that i-line exposure doses in excess of $600 \mathrm{~mJ} / \mathrm{cm}^{2}$ (approximately 10X the recommended dose), were required in combination with baking times of greater than 1 hour at $80^{\circ} \mathrm{C}$ to achieve best adhesion results. Baking temperatures were limited to $80^{\circ} \mathrm{C}$ to prevent plastic deformation of the acrylic. SU-8 features must remain on acrylic after silicone demolding from overhanging features, requiring very large interfacial adhesion strengths if the fibers are to survive multiple demold cycles.

To calibrate the dose provided by the custom UV source, the developed depth of acrylic was measured for different exposures doses from a commercial $254 \mathrm{~nm}$ source. The doses correspond to a $0,5,10$ and 15 hour exposure at a nominal intensity of 4 $\mathrm{mW} / \mathrm{cm}^{2}$ in a Stratalinker 2400. The resulting depths vs. development time are shown in Figure 3 and the unexposed acrylic dissolved at approximately $60 \mathrm{~nm}$ per minute. Because this development rate is highly dependent on the average molecular weight of the acrylic these calibration curves are not valid for all commercial acrylics but high consistency is usually obtained for substrates cut from any individual sheet of OPTIX ${ }^{\circledR}$.

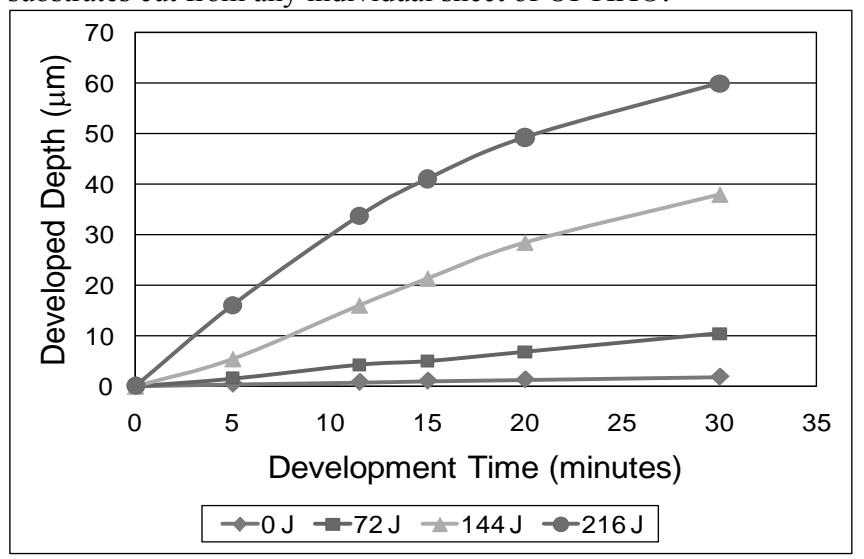

Figure 3. Developed depth of OPTIX® in $\mathrm{SU}-8$ developer at $18^{\circ} \mathrm{C}$ with different exposure doses of uncollimated $254 \mathrm{~nm}$ light. Depth measurements are on wide open areas - uncollimated light will produce smaller depths between tightly spaced features.

The developed depth vs. time can be used to calibrate the approximate dose received by samples exposed in the custom source using different anti-scatter grid designs. Direct comparison between the two grid designs revealed that the 2:1 aspect ratio grid transmitted approximately $35 \%$ of the power compared with a $1: 1$ aspect ratio grid - requiring $3 \mathrm{X}$ the exposure time to achieve similar development rates. Fibers defined with a 1:1 aspect ratio grid had lower true fiber heights than those defined by a 2:1 grid with the same measured depth because a plateau would form under 
closely spaced fibers, as shown in Figure 4. Depending on the dose, the individual fiber heights could be $1 / 2-2 / 3$ the total depth in wide open areas for 1:1 aspect ratio grid exposures.

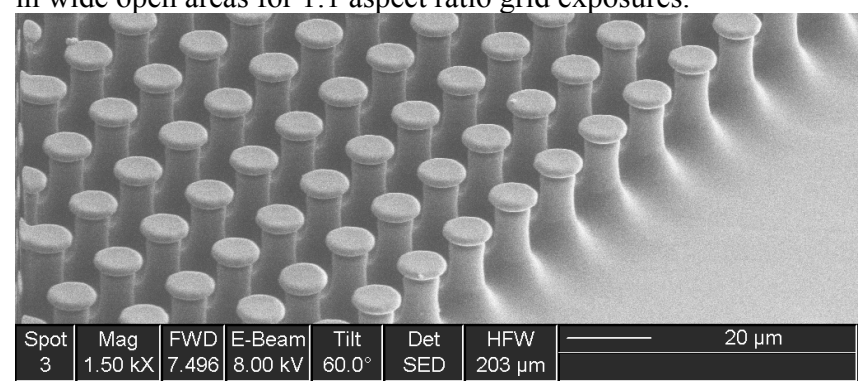

Figure 4. Fibers cast from a mold defined with a 1:1 aspect ratio grid. The fiber base is higher than the base of the open areas.

All fibers defined using a $200 \mathrm{~J}$ exposure dose through the 1:1 grid failed at development times longer than 10 minutes. These fibers were cut off before sufficient undercutting of the cap occurred, making this choice of exposure time impractical for producing an effective adhesive structure. For all other conditions, yield was primarily reduced by caps falling off of the fiber tips during silicone demolding. This was noticeably worse around the perimeter of the 5" 5 " acrylic mold. The reduced light intensity at those locations was found to be responsible for the lower SU-8 adhesion to acrylic (Figure 5). This issue was resolved for other molds by increasing i-line exposure dose to $900 \mathrm{~mJ} / \mathrm{cm}^{2}$.

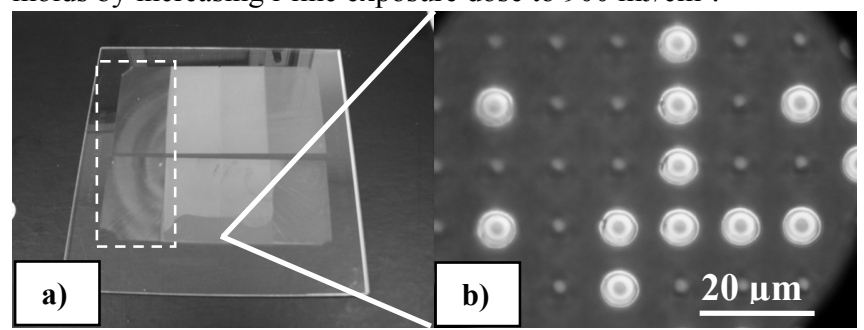

Figure 5. a) 5"x5" acrylic substrate used for tests after a 20 minute development. Cap failure of $6 \mu \mathrm{m}$ diameter features is greatest at the perimeter (b), but fibers are reduced to less than 2 $\mu \mathrm{m}$ in diameter under these conditions. Dashed line indicates where fibers were completely undercut from overexposure.

The geometries that were successfully produced in acrylic and subsequently manufactured in ST-1060 polyurethane were measured using optical methods and scanning electron images of the fibers. The primary dimensions of importance were the cap overhang and fiber height. The adhesion strength increased with increasing cap overhang, while increased fiber height made the dry adhesive designs more effective at conforming to the rounded surface of the hemispherical probe. Because some $254 \mathrm{~nm}$ light does pass through the SU-8 caps, small differences in overhang occur between fibers exposed for different doses at the same development time. The average fiber dimensions are found from profilometer data, SEM and optical images.

Each sample of ST-1060 polyurethane fibers tested for adhesion was $1.5 \mathrm{~mm}$ thick and the pull speed of the hemispherical tip was set to be $5 \mu \mathrm{m} / \mathrm{second}$ for each trial. To save space not every data trial is listed, but a dramatic difference in adhesion strength is observed once the cap overhang becomes slightly larger than its thickness (Figure 6). For ST-1060 fibers with an overhang to thickness ratio greater than $1: 1$, the fiber height has a larger influence, as the adhesive surface can conform better to the hemispherical probe and results in a higher maximum adhesion at larger preloads (Figure 7). All reported data are for the $12 \mu \mathrm{m}$ diameter fiber tip designs defined with a 1:1 aspect ratio grid, which had the highest fabrication yields. Fiber height (including SU-8 cap) and cap overhang in micrometers are listed for each data set.

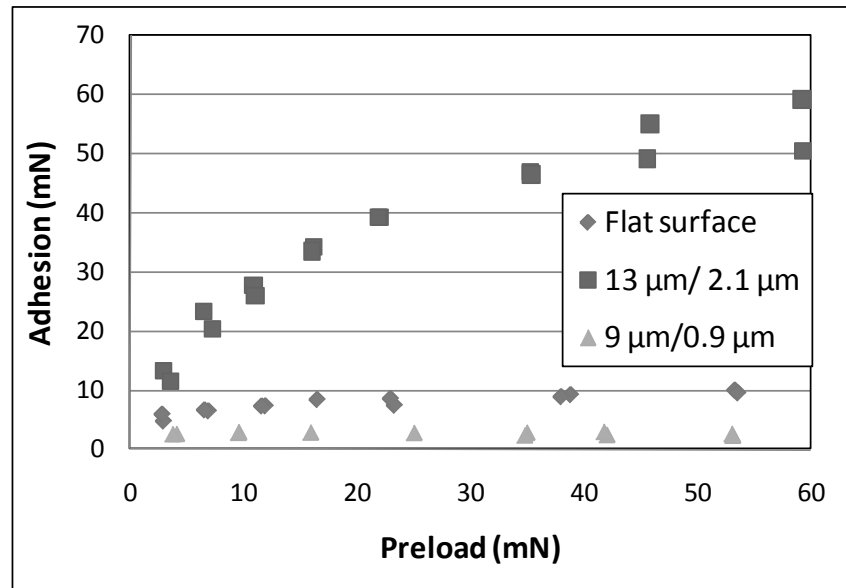

Figure 6. Comparison of adhesion vs. preload for an unstructured ST-1060 surface and fiber height/overhang defined with a 10 and 20 minute development. Exposure dose was approximately $120 \mathrm{~J}$ through a 1:1 aspect ratio grid.

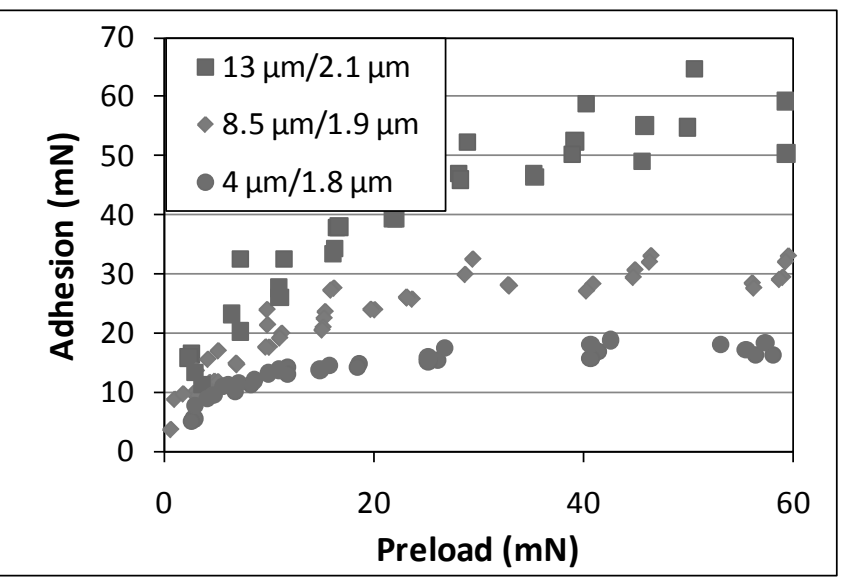

Figure 7. Typical adhesion data for ST-1060 polyurethane fibers defined with different geometries tested at different locations. 254 $\mathrm{nm}$ dose of 120, 80 and $40 \mathrm{~J}$ though a 1:1 grid was used with a development time of 20 minutes

Although the fill factor of each design was identical at approximately $28 \%$, very large differences in adhesion performance are observed for different fiber geometries. As seen in Figure 6, as the cap overhang increased from 0.9 to $1.9 \mu \mathrm{m}$ the adhesion performance changed from worse to much better than an unstructured surface processed in the same manner. Figure 7 shows that there is much more variability in adhesion, but also a higher maximum adhesion value reached for taller fibers with similar cap dimensions. There is an upper limit to this effect however, as still taller fibers will have greatly reduced adhesion as they collapse immediately after demolding or during testing. This was seen primarily with over developed fibers and those defined with equal doses through $2: 1$ aspect ratio grids.

In comparison to the more flexible ST-1060, both higher aspect ratio fibers and larger cap overhangs can be successfully demolded using WC-755. A sample of WC-755 cast from a mold defined using a $100 \mathrm{~J}, 1: 1$ exposure and 40 minute development is 
shown in Figure 8. This geometry was not appropriate for ST1060, but good adhesion and yield was achieved for WC-755 fibers. Qualitative tests indicated that while the structured WC755 would show much greater normal and peel strength on flat surfaces than its unstructured state, adhesion to rounded surfaces like those on our test system was less effective. Plotting preload vs. adhesion strength for this material does not provide an accurate assessment of the performance because the viscoelasticity of the WC-755 was much greater than that of the ST-1060. While the maximum adhesion force of WC-755 did not always increase as dramatically as ST-1060 compared to its unstructured state, the energy dissipated (from the area under the force/displacement curve) was far higher for the structured WC-755 (Figure 9). The uneven adhesion force with displacement is suspected to be due to individual fibers losing contact intermittently. If higher preloads were used, WC-755 fibers would remain deformed for at least a few minutes, and occasionally would not recover.

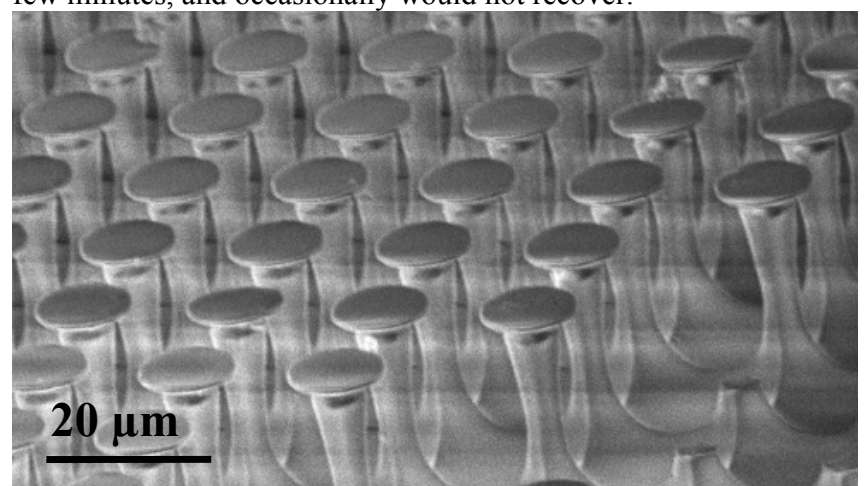

Figure 8. SEM images of WC-755 polyurethane fibers tested for adhesion. Height is approximately $30 \mu \mathrm{m}$ and minimum fiber diameter is approximately $5 \mu \mathrm{m}$.

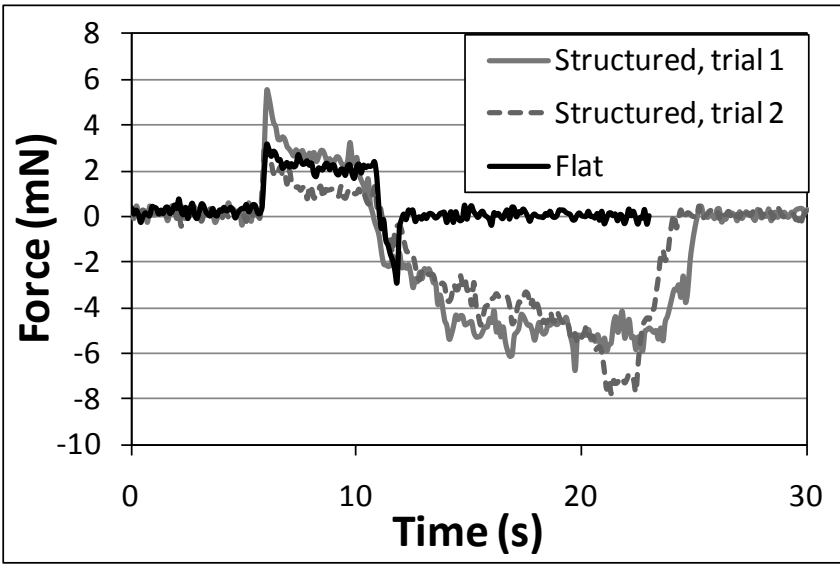

Figure 9. Two individual adhesion trials of the structured WC-755 fibers shown in figure 8 and a flat control. A large reduction in preload force is seen after first contact, demonstrating viscoelasticity.

\section{CONCLUSIONS}

We have developed a novel and unique method of producing biomimetic dry adhesives in a variety of materials and geometries. Using a bulk acrylic substrate can dramatically lower the cost per area of producing dry adhesive structures, and direct photopatterning of acrylic allows a very large variety of fiber geometries to be produced using different process parameters. For low modulus polyurethanes like ST-1060, an anti-scatter grid with a 1:1 aspect ratio is sufficient to make effective dry adhesives.
While a 2:1 aspect ratio grid can produce higher aspect ratio fibers, there is a diminishing return past this point due to the severe reduction in light intensity. These higher aspect ratio fibers are required for stiff materials, but switching to a lower exposure dose and longer development time can also produce the narrow fiber, large cap overhang structures necessary to make stiffer polyurethanes behave as effective dry adhesives. Our primary focus now is on reducing the minimum feature size while maintaining high yield, and finding the best structural materials for long lifetime dry adhesives. The fabrication process developed in this work is very promising for large scale commercial structuring of dry adhesives and other biomimetic materials. Our long term goals will be to determine optimal fiber geometries for synthetic dry adhesives produced in a variety of materials and develop practical design rules for manufacturing these structures.

\section{ACKNOWLEDGEMENTS}

We would like to thank Marius Haiducu and Dr. Ian G. Foulds for the construction of the custom UV source. Thanks are also extended to Dr. M. Parameswaran and the IMMR group at Simon Fraser University for use of their fabrication facilities and equipment. This work was financially supported by NSERC.

\section{REFERENCES}

[1] S. Kim and M. Sitti, "Biologically inspired polymer microfibers with spatulate tips as repeatable fibrillar adhesives," Applied Physics Letters, vol. 89, pp. 261911 1-3, 2006.

[2] H. E. Jeong, et al., "A non transferring dry adhesive with hierarchical polymer nanohairs," Proc. Nat. Acad. Sciences, vol. 106, pp. 5639-5644, 2009.

[3] D. Sameoto and C. Menon, "A low-cost, high yield fabrication method for producing optimized biomimetic dry adhesives," J. Micromech. Microeng., vol. 19, p. 115002 , 2009.

[4] D. Sameoto and C. Menon, "Direct molding of dry adhesives with anisotropic peel strength," J. Micromech. Microeng., vol. 19, p. 115026, 2009.

[5] A. del Campo, et al., "Contact shape controls adhesion of bioinspired fibrillar surfaces," Langmuir, vol. 23, pp. 1023510243, 2007.

[6] M. Murphy, et al., "Adhesion and Anisotropic Friction Enhancements of Angled Heterogeneous Micro-Fiber Arrays with Spherical and Spatula Tips," Journal of Adhesion Science and Technology, vol. 21, pp. 1281-1296, 2007.

[7] B. Aksak, et al., "Gecko inspired micro-fibrillar adhesives for wall climbing robots on micro/nanoscale rough surfaces," IEEE International Conference on Robotics and Automation, pp. 3058-3063, 2008.

[8] R. W. Johnstone, et al., "Deep-UV exposure of poly(methyl methacrylate) at $254 \mathrm{~nm}$ using low-pressure mercury vapor lamps," Journal of Vacuum Science and Technology B: Microelectronics and Nanometer Structures, vol. 26, pp. 682$285,2008$.

[9] R. W. Johnstone, et al., "Self-sacrificial surface micromachining using poly(methyl methacrylate)," $J$. Micromech. Microeng., vol. 18, p. 115012, 2008.

[10] M. Haiducu, et al., "Deep UV patterning of commercial grade PMMA for low cost, large scale microfluidics," $J$. Micromech. Microeng., vol. 18, p. 115029, 2008.

\section{CONTACT}

*D. Sameoto, tel: +1-780-248-2052; sameoto@ualberta.ca 